\title{
Relationship between MGMT gene expression and treatment effectiveness and prognosis in glioma
}

\author{
QIANG LI ${ }^{1}$, JIANG GUO ${ }^{2}$, WEISHENG WANG ${ }^{3}$ and DINGKUN WANG ${ }^{4}$ \\ ${ }^{1}$ Department of Neurosurgery, Laiwu City People's Hospital, Laiwu, Shandong 271100; \\ ${ }^{2}$ Department of Neurosurgery, Shouguang City People's Hospital, Shouguang, Shandong 262100; \\ ${ }^{3}$ Department of Neurology, Liaocheng Third People's Hospital, Liaocheng, Shandong 252000; \\ ${ }^{4}$ Department of Neurosurgery, Tongde Hospital of Zhejiang, Hangzhou, Zhejiang 310012, P.R. China
}

Received November 17, 2016; Accepted January 31, 2017

DOI: $10.3892 / \mathrm{ol} .2017 .6123$

\begin{abstract}
The expression of $\mathrm{O}^{6}$-methylguanine DNA methyltransferase (MGMT) in different grade gliomas were analyzed in relation to its therapeutic effect and impact on disease prognosis. In total, 62 patients with glioma, who were admitted by neurosurgery and received surgical treatment and postoperative conventional chemoradiation, were selected for this study. Expression of MGMT was greater with an increase in brain glioma grade. Gender, age, tumor size and Karnofsky performance status (KPS) score did not differ with MGMT expression $(\mathrm{P}>0.05)$. Expression of MGMT in normal brain tissue was slightly significantly different than expression of MGMT in glioma tissue $(\mathrm{P}<0.05)$. The short-term efficacy and survival time of the MGMT-negative expression group were better than those of MGMT-positive expression. MGMT was only treated as an index to monitor tumor recurrence or metastasis and a reference to judge the prognosis of patients. The expression level of MGMT in glioma had no relation with age, gender, tumor size, surgical approach and KPS score. For glioma patients with positive expression of MGMT, antineoplastic drugs of alkylating agent class should be avoided.
\end{abstract}

\section{Introduction}

Glioma is not only the most common primary intracranial tumor (1), but also the most intractable in the treatment of neurosurgery tumors. Incidence rates of about 5 in 100,000 have been reported with a rising trend (2). The treatment of glioma has shown great improvement for patients who are diagnosed early with excision often used as treatment approach (3). However, surgical treatment has a poor effect for the malignant growth of tumor cells, leading to high recurrence and mortality rates (4). In order to decrease the recurrence and mortality rate, patients

Correspondence to: Dr Dingkun Wang, Department of Neurosurgery, Tongde Hospital of Zhejiang, 234 Gucui Road, Hangzhou, Zhejiang 310012, P.R. China

E-mail: shkbkdy3182@163.com

Key words: glioma, MGMT, resistance gene receive antineoplastic drugs post-operatively (5). However, data show that patients diagnosed with glioma, have a survival time of only $12-15$ months, and the 5-year survival rate $<5 \%$ (6).

$\mathrm{O}^{6}$-methylguanine DNA methyltransferase (MGMT) is a DNA repair protein, found in humans and many prokaryotic organisms. Research has shown that for the patients with glioma, antineoplastic drug treatments by alkylating agents may result in drug resistance, and MGMT is the main reason and therefore, not an ideal treatment for patients (7). Some reports indicate that the effect of positive MGMT expression is inferior to that of negative expression during chemotherapy for the patient with glioma (8). Thus, MGMT activity is higher, and the drug resistance to antineoplastic drugs of alkylating agent class is greater. If MGMT expression is lower, the alkylating class of antineoplastic drugs is more sensitive, with a better efficacy. We analyzed the expression of MGMT in newly diagnosed glioma patients to increase the sensitivity of patients with glioma to antineoplastic drugs of alkylating agent class so as to significantly improve the prognosis and reduce the reoccurrence.

\section{Materials and methods}

In the study, 62 patients with glioma, who were admitted by neurosurgery from January 2011 to January 2013, were selected. Post-surgical treatment was required and the pathology results were diagnosed as glioma. A total of 33 males (aged 25-72 years; average 42.7 years) and 29 females (aged 23-71 years; average 40.1 years) were included. There were 15 cases of grade I, 16 cases of grade II, 14 cases of grade III and 17 cases of grade IV glioma. As a control group we used brain tissue from 12 patients with cerebral hemorrhage caused by high blood pressure (aged 40-55 years; average 48.1 years). It was required that the time between incidence and operative treatment was not more than $6 \mathrm{~h}$.

This study was approved by the Ethics Committee of Tongde Hospital of Zhejiang Province. Signed written informed consents were obtained from all participants before the study.

Inclusion criteria. In the study were: i) Age $\geq 18$ years old and Karnofsky performance status (KPS) $>60$ scores; ii) women 
Table I. MGMT distribution in different grades of glioma.

\begin{tabular}{lrrrrr}
\hline $\begin{array}{l}\text { MGMT } \\
\text { expression }\end{array}$ & I & II & III & IV & Total \\
\hline- & 10 & 8 & 6 & 5 & 29 \\
+ & 5 & 5 & 2 & 5 & 17 \\
++ & 0 & 3 & 4 & 4 & 11 \\
+++ & 0 & 0 & 2 & 3 & 5 \\
Total & 15 & 16 & 14 & 17 & 62 \\
\hline
\end{tabular}

MGMT, $\mathrm{O}^{6}$-methylguanine DNA methyltransferase.

of non-gestation or non-lactation; iii) normal heart, brain, liver, lung and kidney; iv) all patients received surgical treatment of tumor resection, and the postoperative pathological diagnosis results could be confirmed; v) complications such as intracranial infection and intracranial hematoma did not occur after operation; vi) favorable paraffin-embedded tissue; and vii) patients with good compliance, complete data of cases and coordination of follow-up.

Therapeutic regimen. All patients had surgical treatment, and the tumor was resected as much as possible on the precondition of retaining neurological function. In the study, 62 patients were given chemotherapy combining with radiotherapy after operation. After 2-3 weeks, the patients were given conformal radiotherapy of photon knife (Elekta, Stockholm, Sweden), with at least one course of treatment. On the third day of radiotherapy, 42-day chemotherapy by the dose of $75 \mathrm{mg} / \mathrm{m}^{2}$ temozolomide (TMZ) (Biosharp, Hefei, China) was started. After 4 weeks of combination of chemotherapy and radiotherapy, for 2-periods a single-drug chemotherapy of trimethylamine (TMA) was carried out. During the process of chemoradiotherapy, if the patients had encephaledema, intracranial pressure was decreased by dehydration and diuresis treatments. Blood routine examination was reviewed once a week, and hepatorenal function was tested at fixed period. Symptomatic treatment such as liver protection also was conducted according to the test situation and clinical symptoms.

Immunohistochemical methods. SP immunohistochemical method was applied and the steps proceeded according to instructions. In cell nucleus and/or intracytoplasm, the yellow or claybank indicated positive MGMT. The specific judgement methods of positive MGMT were as follows: under the scope of high power lens (400 times), 10 representative areas that gathered glioma cells were selected, the rate of positive cells was observed, which was divided into four grades: i) Negative (-), without positive cells or $<10 \%$; ii) weakly positive $(+)$, positive cells among 10 scopes of $10-24 \%$; iii) intermediately positive (++), positive cells of $25-50 \%$; and iv) strongly positive $(+++)$, positive cells $>50 \%$.

Observation methods. The time from initial chemotherapy to the last return visit, or death was tracked, namely, the overall

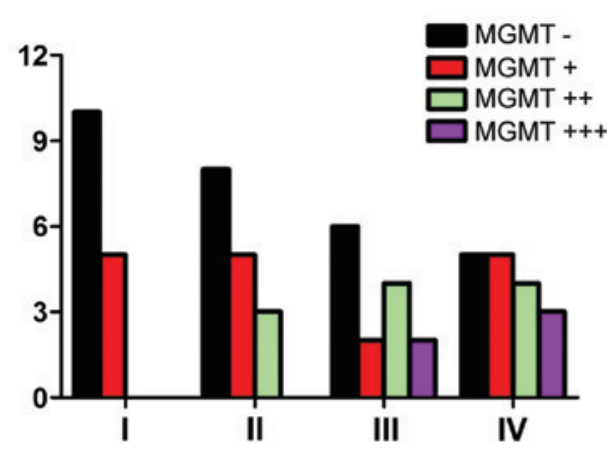

Figure 1. MGMT distribution in different grades of glioma. MGMT, $\mathrm{O}^{6}$-methylguanine DNA methyltransferase.

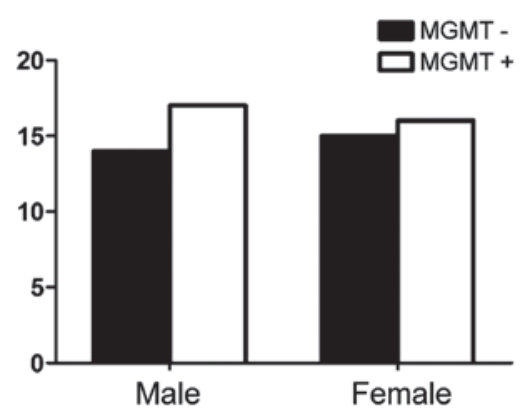

Figure 2. Relationship between MGMT expression and gender. MGMT, $\mathrm{O}^{6}$-methylguanine DNA methyltransferase.

survival (OS). Related data were recorded and Kaplan-Meier survival curve was drawn. The level of myelosuppression was determined by WHO grade. According to the subjective feelings and clinical manifestation of patients, gastrointestinal symptoms were judged.

Statistical analysis. SPSS 16.0 (SPSS, Inc., Chicago, IL, USA) was used to analyze the data. The $\chi^{2}$ test was applied to detect the difference of positive rate for different grades of glioma. $\mathrm{P}<0.05$ meant that the difference had statistical significance. Fisher's exact probability test was used to judge the relation between MGMT expression and gender and age for the patients with glioma. Spearman's correlation was taken to analyze the expression of MGMT in different grades of glioma; Kaplan-Meier survival curve presented the relation between the survival time and of the patient tissues, and log-rank detection was also conducted at the same time.

\section{Results}

MGMT expression in different grades of glioma. As shown in Table I and Fig. 1, the negative expression of MGMT reached $66.7 \%$ in grade I, $50 \%$ in grade II, $42.9 \%$ in grade III and 29.4\% in grade IV. MGMT expression increased in high-grade glioma, and the expression gradually decreased in low-grade glioma. By statistical analysis, it was confirmed that the expression of MGMT had no relation with WHO grade $(\mathrm{P}>0.05)$.

MGMT expression and biological characteristics. Fig. 2 shows the expression of MGMT among males was $52.9 \%$, 


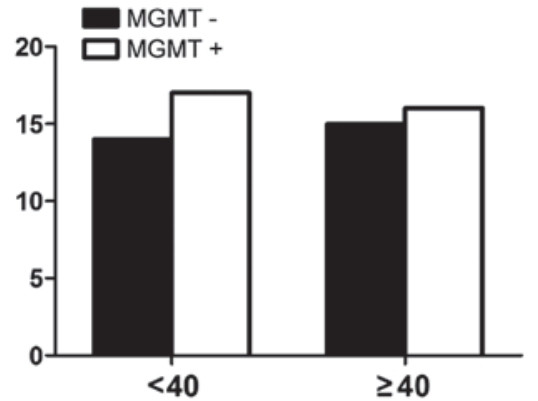

Figure 3. Relationship between MGMT expression and age. MGMT, $\mathrm{O}^{6}$-methylguanine DNA methyltransferase.

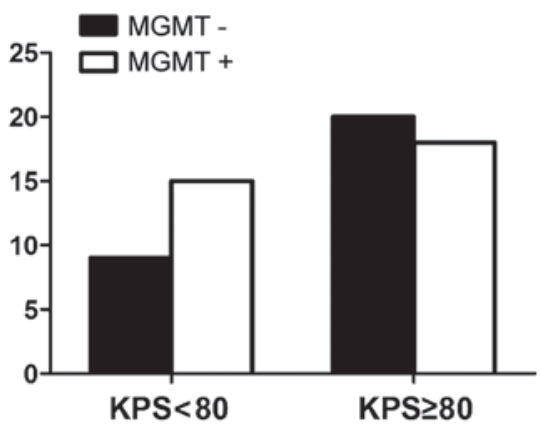

Figure 4. Relationship between MGMT expression and pre-operative KPS score. MGMT, $\mathrm{O}^{6}$-methylguanine DNA methyltransferase; KPS, Karnofsky performance status.

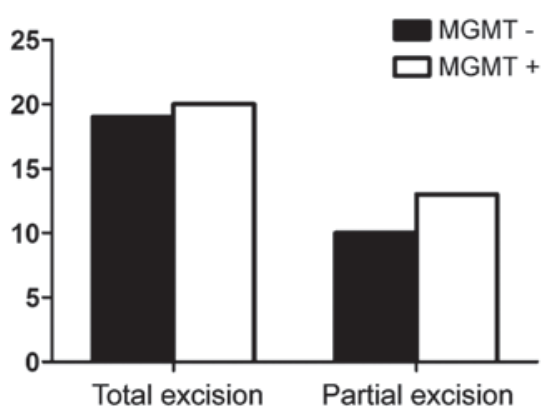

Figure 5. Relationship between MGMT expression and excision method. MGMT, $\mathrm{O}^{6}$-methylguanine DNA methyltransferase.

while the positive expression of MGMT among females was $53.6 \%(\mathrm{P}>0.05)$ and no correlation with age (Fig. 3 ). There were nine cases with negative expression of MGMT in patients whose KPS score was $<80$ and 20 cases in patients whose score was $>80$ ( $\mathrm{P}>0.05)$ (Fig. 4). The expression of MGMT was not related to the method of excision $(\mathrm{P}>0.05)$ (Fig. 5). In patients whose tumor volume was $<50 \mathrm{~cm}^{3}, 44.8 \%$ had negative and $55.2 \%$ had positive expression rate and the difference had no statistical significance $(P>0.05)$ (Fig. 6). The negative expression in low-grade glioma (grade I and II) was $58.1 \%$ and the positive rate was $41.9 \%$, while the negative expression in high-grade (grade III and IV) was $35.5 \%$ and the positive expression was $54.5 \%$ (Fig. 7). The positive expression of MGMT in low-grade glioma was significantly lower than that of high-grade $(\mathrm{P}<0.05)$. However, gender, age, tumor size, surgical method and KPS score had no

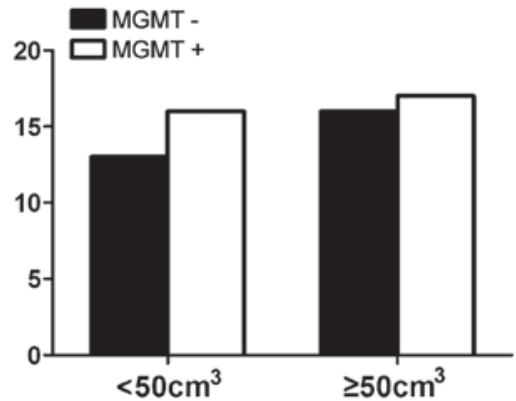

Figure 6. Relationship between MGMT expression and tumor volume. MGMT, $\mathrm{O}^{6}$-methylguanine DNA methyltransferase.

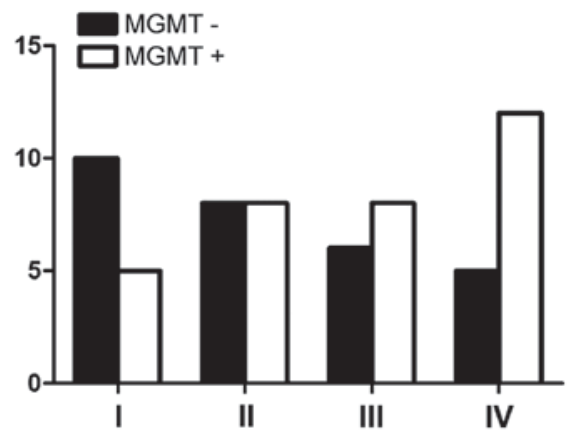

Figure 7. Positive expression of MGMT in different grades. MGMT, $\mathrm{O}^{6}$-methylguanine DNA methyltransferase.

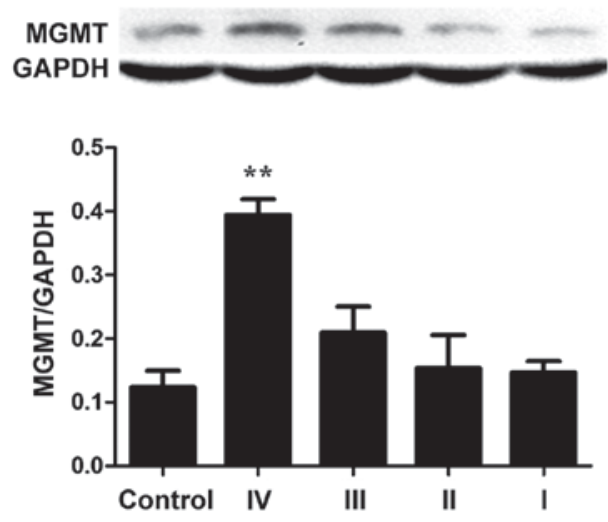

Figure 8. Protein expression of MGMT in different grades. MGMT, $\mathrm{O}^{6}$-methylguanine DNA methyltransferase. ${ }^{* *} \mathrm{P}<0.05$ compared to control.

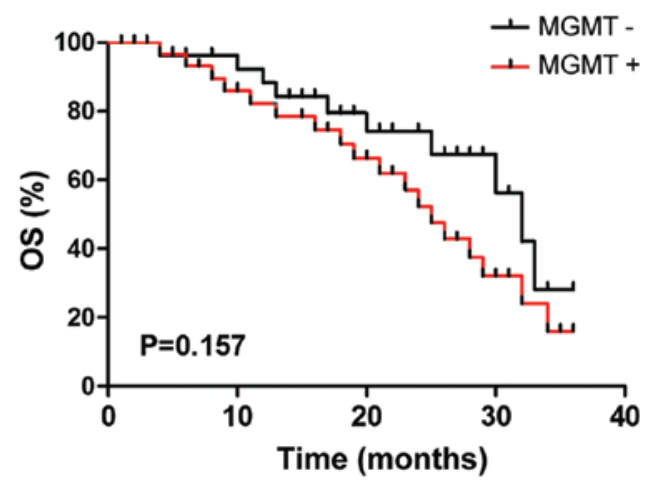

Figure 9. Comparison of the patient survival curve between MGMT negative expression group and MGMT positive expression group. MGMT, $\mathrm{O}^{6}$-methylguanine DNA methyltransferase. 
statistical difference with MGMT among the 62 patients $(\mathrm{P}>0.05)$.

Western blotting. Protein expression of MGMT in normal controls was the lowest, while protein expression increased with increasing glioma grade especially for grade IV (Fig. 8). The expression of MGMT was 3.2-fold that of the control group, and the difference had statistical significance $(\mathrm{P}<0.01)$, but the expression of each tumor grade showed no relation with WHO tumor grade $(\mathrm{P}>0.05)$.

Evaluation of short-term remission. After treatment, the patients were divided into complete remission and partial remission as according to the short-term evaluation standard of WHO. In this experiment, the objective efficacy for patients with negative MGMT protein expression was better than that of MGMT-positive expression group. In MGMT negative expression group, the effective rate reached $72.4 \%(21 / 29)$, while the effective rate of MGMT-positive expression group was only $18.2 \%$ (5/33), with statistical significance $(\mathrm{P}<0.05)$.

Survival time. The 3-year Kaplan-Meier survival of patients with MGMT-negative expression was 65.5\% (19 cases), while the survival rate of patients with MGMT-positive expression was $45.5 \%$ ( 15 cases), but the difference had no statistical significance by log-rank detection $(\mathrm{P}>0.05)$ (Fig. 9).

\section{Discussion}

Glioma often occurs in the neurogliocyte of neuroderm, which is a most common malignant tumor with high recurrence, fatality and low recovery rates. The tumor cell has obvious effect, high invasiveness and infiltrative growth. Tumor cell proliferation is fast and expands to other brain tissue. Currently, the main therapy for glioma is a comprehensive treatment including neurosurgical operation, chemotherapy and radiotherapy. However, the prognosis is not ideal (9-11).

Radiotherapy is a vital step in the process of glioma treatment, and the main chemotherapeutic agent is from the alkylating class of drugs. These drugs can alkylate DNA and form crosslinks, leading to high cytotoxicity, effect on DNA replication leading to tumor cell death. MGMT is distributed in different tissues, with brain tissue among the lowest. The expression of MGMT in normal tissue is lower than that of tumor tissue, whose activity is often taken as an important index for observing the prognosis of patients. MGMT can act on DNA crosslinks and restore DNA alkylation, reducing the toxic effect of alkane chemotherapy drugs and causing MGMT inactivation, so that patients develop drug resistance. As a result, the expression level of MGMT can influence the drug resistance of an alkylating agent in tumor cells (12). However, besides individual variation, the main reason that influences the chemotherapeutic effect for tumor also depends on drug resistance (13). The survival time of the patients with glioma is usually only $12-15$ months, up to $90 \%$ of patients die due to drug tolerance of the tumor cells (14). Therefore, before the treatment on glioma, it is important to identify high expression of MGMT for the chemotherapy prognosis of patients. For the patients with glioma, the current first line of chemotherapeutics is alkylating agent TMZ and new nitrosourea drugs. Some clinical research has confirmed that the treatment effect of radiotherapy combined with TMZ was better than that of single radiotherapy for patients with glioma who received tumor excision, which can increase the survival rate of patients from 10 to $26 \%$ and improve disease prognosis (15).

In this study, gender, age, tumor size, surgical method and KPS score had no statistical difference in MGMT expression. This suggests that MGMT could not be used as an independent reference for the individual chemotherapy regimen in clinic. At the same time, it was confirmed that the difference between positive expression of MGMT and WHO grade of glioma had statistical significance. The expression of MGMT in glioma tissue was higher than that of normal tissue and with an increase in WHO grade, the positive expression of MGMT was higher. Research by Yuan et al showed that expression of MGMT in the patients with lower level malignancy increased, while the expression was decreased in the patients with high-grade malignancy (16-18). Other research has also confirmed that there was no obvious correlation between the positive expression of MGMT and WHO grade (18). However, the conclusions were based on limited cases.

Our results show that the survival time of the MGMTnegative expression group was longer than that of MGMT-positive expression group, but the difference showed no statistical significance by log-rank detection. It suggests that for glioma patients with positive expression of MGMT, antineoplastic drugs of alkylating agent class should be avoided, so as to provide patients with a better personalized treatment (19-21).

\section{References}

1. Blumenthal DT, Dvir A, Lossos A, Tzuk-Shina T, Lior T, Limon D, Yust-Katz S, Lokiec A, Ram Z, Ross JS, et al: Clinical utility and treatment outcome of comprehensive genomic profiling in high grade glioma patients. J Neurooncol 130: 211-219, 2016.

2. Stupp R, Hegi ME, van den Bent MJ, Mason WP, Weller M, Mirimanoff RO and Cairncross JG; European Organisation for Research and Treatment of Cancer Brain Tumor and Radiotherapy Groups; National Cancer Institute of Canada Clinical Trials Group: Changing paradigms - an update on the multidisciplinary management of malignant glioma. Oncologist 11: 165-180, 2006.

3. Kaina B, Christmann M, Naumann S and Roos WP: MGMT: Key node in the battle against genotoxicity, carcinogenicity and apoptosis induced by alkylating agents. DNA Repair (Amst) 6: 1079-1099, 2007.

4. Hsu CY, Lin SC, Ho HL, Chang-Chien YC, Hsu SP, Yen YS, Chen MH, Guo WY and Ho DM: Exclusion of histiocytes/endothelial cells and using endothelial cells as internal reference are crucial for interpretation of MGMT immunohistochemistry in glioblastoma. Am J Surg Pathol 37: 264-271, 2013.

5. Jacinto FV and Esteller M: MGMT hypermethylation: A prognostic foe, a predictive friend. DNA Repair (Amst) 6: 1155-1160, 2007.

6. Bocangel D, Sengupta S, Mitra S and Bhakat KK: p53-mediated down-regulation of the human DNA repair gene $O^{6}$-methylguanine-DNA methyltransferase (MGMT) via interaction with Sp1 transcription factor. Anticancer Res 29: 3741-3750, 2009.

7. Molnár J, Engi H, Hohmann J, Molnár P, Deli J, Wesolowska O, Michalak $\mathrm{K}$ and Wang Q: Reversal of multidrug resitance by natural substances from plants. Curr Top Med Chem 10: 1757-1768, 2010.

8. Tugcu B, Postalci LS, Gunaldi O, Tanriverdi O and Akdemir H: Efficacy of clinical prognostic factors on survival in patients with glioblastoma. Turk Neurosurg 20: 117-125, 2010. 
9. Yoshino A, Ogino A, Yachi K, Ohta T, Fukushima T, Watanabe T, Katayama Y, Okamoto Y, Naruse N, Sano E, et al: Gene expression profiling predicts response to temozolomide in malignant gliomas. Int J Oncol 36: 1367-1377, 2010.

10. Lombardi G, Pace A, Pasqualetti F, Rizzato S, Faedi M, Anghileri E, Nicolotto E, Bazzoli E, Bellu L, Villani V, et al: Predictors of survival and effect of short (40 Gy) or standard-course $(60 \mathrm{~Gy})$ irradiation plus concomitant temozolomide in elderly patients with glioblastoma: A multicenter retrospective study of AINO (Italian Association of Neuro-Oncology). J Neurooncol 125: 359-367, 2015.

11. Suryadevara CM, Verla T, Sanchez-Perez L, Reap EA, Choi BD, Fecci PE and Sampson JH: Immunotherapy for malignant glioma. Surg Neurol Int 6 (Suppl 1): S68-S77, 2015.

12. Chen ZP, Malapetsa A, McQuillan A, Marcantonio D, Bello V, Mohr G, Remack J, Brent TP and Panasci LC: Evidence for nucleotide excision repair as a modifying factor of $O^{6}$-methylguanine-DNA methyltransferase-mediated innate chloroethylnitrosourea resistance in human tumor cell lines. Mol Pharmacol 52: 815-820, 1997.

13. Stupp R, Hegi ME, Gilbert MR and Chakravarti A: Chemoradiotherapy in malignant glioma: Standard of care and future directions. J Clin Oncol 25: 4127-4136, 2007.

14. Bush ZM, Longtine JA, Cunningham T, Schiff D, Jane JA Jr, Vance ML, Thorner MO, Laws ER Jr and Lopes MB: Temozolomide treatment for aggressive pituitary tumors: Correlation of clinical outcome with $\mathrm{O}^{6}$-methylguanine methyltransferase (MGMT) promoter methylation and expression. J Clin Endocrinol Metab 95: E280-E290, 2010
15. Nitta M, Muragaki Y, Maruyama T, Ikuta S, Komori T, Maebayashi K, Iseki H, Tamura M, Saito T, Okamoto S, et al: Proposed therapeutic strategy for adult low-grade glioma based on aggressive tumor resection. Neurosurg Focus 38: E7, 2015.

16. Yuan Q, Matsumoto K, Nakabeppu Y and Iwaki T: A comparative immunohistochemistry of $\mathrm{O}^{6}$-methylguanine-DNA methyltransferase and p53 in diffusely infiltrating astrocytomas. Neuropathology 23: 203-209, 2003.

17. Esteller M, Garcia-Foncillas J, Andion E, Goodman SN, Hidalgo OF, Vanaclocha V, Baylin SB and Herman JG: Inactivation of the DNA-repair gene MGMT and the clinical response of gliomas to alkylating agents. N Engl J Med 343: 1350-1354, 2000

18. Rimel BJ, Huettner P, Powell MA, Mutch DG and Goodfellow PJ: Absence of MGMT promoter methylation in endometrial cancer. Gynecol Oncol 112: 224-228, 2009.

19. Lavon I, Zrihan D, Zelikovitch B, Fellig Y, Fuchs D, Soffer D and Siegal T: Longitudinal assessment of genetic and epigenetic markers in oligodendrogliomas. Clin Cancer Res 13: 1429-1437, 2007.

20. Bobola MS, Blank A, Berger MS and Silber JR: $\mathrm{O}^{6}$-methyl guanine-DNA methyltransferase deficiency in developing brain: Implications for brain tumorigenesis. DNA Repair (Amst) 6: 1127-1133, 2007.

21. Rapkins RW, Wang F, Nguyen HN, Cloughesy TF, Lai A, Ha W, Nowak AK, Hitchins MP and McDonald KL: The MGMT promoter SNP rs16906252 is a risk factor for MGMT methylation in glioblastoma and is predictive of response to temozolomide. Neuro Oncol 17: 1589-1598, 2015. 